\title{
Shades of gray between large B-cell lymphomas and Hodgkin lymphomas: differential diagnosis and biological implications
}

\author{
Nancy L Harris \\ Department of Pathology, Massachusetts General Hospital and Harvard Medical School, Boston, MA, USA
}

\begin{abstract}
Hodgkin lymphomas (HLs) are neoplasms of large B cells. Two types are recognized: nodular lymphocyte predominant HL (NLPHL) and classical HL (CHL). In both types, there may be morphological and possibly biological overlap with large B-cell lymphomas (LBCLs) of non-HL types. These include nodular sclerosis CHL and primary mediastinal large B-cell lymphoma; CHL rich in lymphocytes and NLPHL; and NLPHL and T-cell/ histiocyte-rich LBCL. This review covers the defining features of each of these diseases, the borderlines between them, and strategies for differential diagnosis.
\end{abstract}

Modern Pathology (2013) 26, S57-S70; doi:10.1038/modpathol.2012.182

Keywords: classification; immunophenotype; lymphoma

In the 1990s, it was recognized that the neoplastic cells of Hodgkin lymphoma (HL), both classical HL (CHL) and nodular lymphocyte predominant HL (NLPHL) were of B-cell lineage. This finding clearly justified the inclusion of HL with the so-called 'nonHodgkin' lymphomas (NHLs) in the Revised European-American Classification of Lymphoid Neoplasms (REAL Classification) and the subsequent World Health Organization Classifications of Hematopoietic and Lymphoid Neoplasms (WHO classification). ${ }^{1,2}$ In addition, it opened the door to the idea that borderline (or so-called gray-zone) lymphomas with features of both HL and NHL (mainly large B-cell lymphomas (LBCLs)) might exist. Both CHL and NLPHL are characterized by the presence of variable numbers of large malignant $B$ cells in a background of non-neoplastic inflammatory cells. In contrast, most NHLs are comprised predominantly of neoplastic cells, with only a minor component of large cells. However, some cases of HL may have large numbers of neoplastic cells, and some types of LBCL are characterized by a background rich in inflammatory cells; these cases pose a challenge in differential diagnosis and also suggest the possibility of a bridge-or biological overlap-

Correspondence: Dr NL Harris, MD, Massachusetts General Hospital Department of Pathology-Warren 2, 32 Fruit Street, Boston, MA 02114, USA.

E-mail: nlharris@partners.org

Received 4 September 2012; accepted 4 September 2012 between HL and LBCL. The major overlaps occur between nodular sclerosis CHL (NSCHL) and primary mediastinal large B-cell lymphoma (PMBL); CHL rich in lymphocytes (LRCHLs) and NLPHL; and NLPHL and T-cell/histiocyte-rich LBCL (THRLBCL). This review will first discuss CHL and PMBL and their gray zones, followed by NLPHL, THRLBCL and their gray zones.

\section{Classical HL}

CHL is characterized by the presence of characteristic large neoplastic cells: mononuclear Hodgkin cells and binucleated or multinucleated ReedSternberg cells (HRS cells) in a background of variable numbers of lymphocytes, histiocytes, eosinophils and plasma cells (Figures 1a-f). ${ }^{3}$ Diagnostic Reed-Sternberg (RS) cells are large cells with bilobed, double or multiple nuclei, with a large, eosinophilic, inclusion-like nucleolus in at least two lobes or nuclei. The neoplastic cells typically lack conventional markers of the B-cell differentiation program (Figure 2), including pan-B-cell markers such as CD19, CD20, CD79a, and immunoglobulin heavy and light chains, but express the B-cell antigen Pax5 (usually more weakly than normal small B cells). Oct2 and/or Bob1 may be expressed, but both are not typically strongly positive. Some cases have weak and variable expression of CD20. They are typically IRF4/Mum1 + but CD138 - . In 

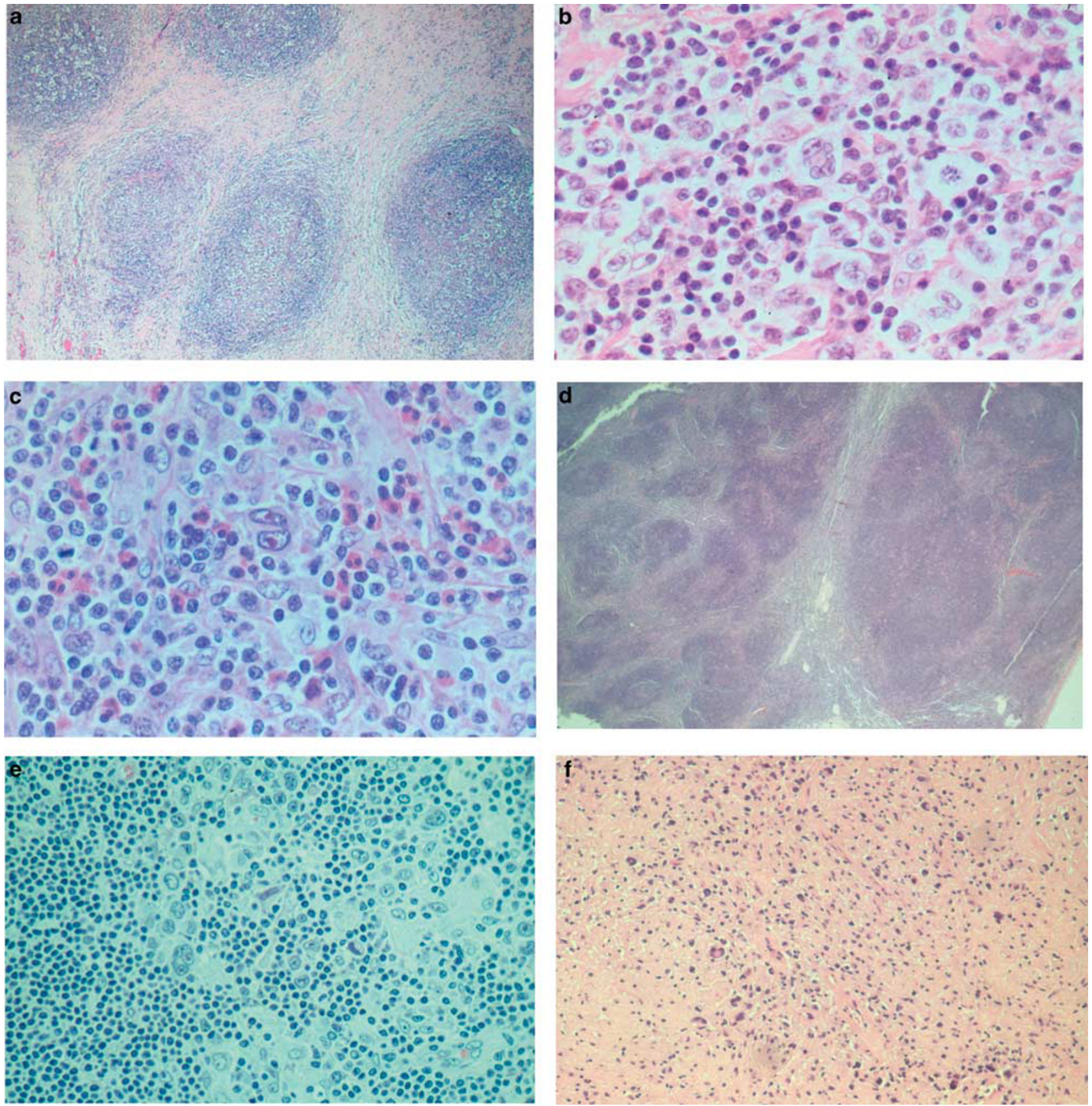

Figure 1 Morphological features of classical Hodgkin lymphoma (CHL) (hematoxylin and eosin stain). (a) Nodular sclerosis CHL (NSCHL) is characterized by cellular nodules surrounded by fibrous bands. (b) The infiltrate contains lacunar variants of Reed-Sternberg (RS) cells, with lobulated nuclei and abundant pale cytoplasm, in a background of lymphocytes and variable numbers of histiocytes and eosinophils. (c) Mixed cellularity CHL (MCCHL) is characterized by classical RS cells and mononuclear variants in a background of lymphocytes, histiocytes and eosinophils. (d) Lymphocyte-rich CHL (LRCHL) has a nodular pattern, with meshworks of follicular dendritic cell (FDC) and sometimes remnants of regressed germinal centers. (e) Classical RS cells and variants are located within the follicles, mantle zones and interfollicular regions in a background of lymphocytes and histiocytes. (f) Lymphocyte-depleted CHL (LDCHL) has a diffuse pattern with variable amounts of sclerosis and often bizarre RS cell variants.

addition, they express a marker not typically associated with normal B cells, CD15, and the activation marker, CD30. The background inflammatory cells typically include $\mathrm{T}$ cells and variable numbers of B cells; the latter may form follicular aggregates with which the neoplastic cells may be associated-a variant known as LRCHL. Immuno- globulin genes (IGH) rearranged and highly mutated. Conventional cytogenetics shows frequent aneuploidy and polyploidy but no consistent recurring abnormalities. CGH has shown gains of $2 p, 9 p$ and $12 q$ and amplification of 4p16, 4q23-24 and 9p23$24 .^{3}$ There is activation of the NFkB, AP-1 and JAK/ STAT pathways in HRS cells, with expression of 
TRAF and nuclear REL. ${ }^{3}$ Recently, translocations of the major histocompatibility complex (MHC) class II transactivator CIITA have been reported in $15 \%$ of the cases of CHL. ${ }^{4}$

Variants of CHL are defined both by the morphology of the neoplastic cells and the characteristics of the background infiltrate, and include nodular sclerosis (NSCHL), ${ }^{5}$ mixed cellularity (MCCHL), ${ }^{6}$ lymphocyte-rich (LRCHL) ${ }^{7}$ and lymphocytedepleted (LDCHL) ${ }^{8}$ types. Particularly in the NSCHL and LDCHL subtypes, large numbers of neoplastic cells may be present and give rise to a differential diagnosis of LBCL.

Distinctive clinical features include the typical presentation of NSCHL in the mediastinum in young adults, with frequent occurrence in females; presentation of MCCHL and LDCHL with advanced disease, splenic and/or bone marrow involvement, and association with immunodeficiency, particularly human immunodeficiency virus (HIV) infection, as well as EBV positivity.

\section{Nodular Sclerosis CHL}

NSCHL is characterized by collagen bands that surround at least one nodule, and HRS cells including some with 'lacunar' type morphology-a multilobated nucleus, small nucleoli, and abundant, pale cytoplasm, which retracts in formalin-fixed sections, producing an empty space or lacuna (Figures 1a and b). The background contains lymphocytes, histiocytes, eosinophils and plasma cells. ${ }^{5}$ Necrosis may be prominent. Histiocytes may form sarcoidal or necrotizing granulomas. In the socalled syncytial variant, there are large sheets of RS cell variants. NS can be stratified according to the number of neoplastic cells or the characteristics of the background infiltrate (proportion of eosinophils) either of which may predict prognosis. However, these grading schemes are not currently used to determine therapy. ${ }^{5}$ EBV positivity is uncommon $(\sim 20 \%)$.

NSCHL is the most common subtype of HL in developed countries (60-80\% in most series). It is most common in adolescents and young adults, but can occur at any age; affected females equal or exceed males. The mediastinum and other supradiaphragmatic sites are commonly involved.

\section{Mixed Cellularity CHL}

In MCCHL, the infiltrate is diffuse or vaguely nodular, without band-forming sclerosis, although fine interstitial fibrosis may be present. RS cells are of the classic, diagnostic type and are usually easily identified (Figure 1c). Many mononuclear variants are usually also present. The infiltrate typically contains lymphocytes, epithelioid histiocytes, eosinophils and plasma cells. EBV positivity is seen in about $75 \%$ of the cases. ${ }^{5}$
MCCHL comprises $15-30 \%$ of HL cases in most series; it may be seen at any age (median age of 40 years), and lacks the early adult peak of NSCHL. Involvement of the mediastinum is less common, and abdominal lymph node and splenic involvement are more common.

\section{Lymphocyte-Rich CHL}

Some cases of CHL have a background infiltrate that consists predominantly of lymphocytes, with rare or no eosinophils (Figures 1d and e). The term LRCHL was proposed for these cases in the REAL classification and was adopted by the WHO. ${ }^{7}$ Most cases of LRCHL have a nodular pattern, with meshworks of follicular dendritic cell (FDC) and remnants of regressed germinal centers; RS cells and variants are located within the mantle zones and interfollicular regions, mimicking NLPHL. However, NLPHL rarely has a distinct germinal center remnant within the nodules.

Immunophenotyping (Figure 2a-c) is essential to distinguish LRCHL from NLPHL, as in LRCHL the RS cells should have a classical (CD20 - CD15 + CD30 + IRF4/Mum1 +) immunophenotype in contrast to the NLPHL, which is typically CD20+ CD15 - CD30 - IRF4/Mum1 - , although exceptions occur. The immunophenotype of the B-cell nodules may be very similar in both entities, including the presence of $\mathrm{T}$ cells expressing CD57 and CD279 associated with the RS cells. ${ }^{9}$ In one study, the B-cell expression program (Oct2, Bob1) was more often preserved in LRCHL compared with other CHL subtypes, and had infrequent expression of REL compared with other CHL but comparable expression of TRAF1, suggesting that LRCHL may be a distinct entity from either CHL or NLPHL. ${ }^{10}$

LRCHL comprises about $5 \%$ of the cases of HL. ${ }^{11}$ The clinical features at presentation similar to those of NLPHL: similar to NLPHL, patients have early stage disease, lack bulky disease or B-symptoms, lack mediastinal disease and have a predominance of males, and a median age higher than that for NSCHL. The prognosis appears to be slightly better than that of other subtypes of CHL, and similar to that of NLPHL, although with fewer late relapses. ${ }^{7,11}$

\section{Lymphocyte-Depleted CHL}

LDCHL is characterized by RS cells, and bizarre 'sarcomatous' variants, and relatively few inflammatory cells. In some cases there is a diffuse hypocellular infiltrate, with diffuse fibrosis and necrosis (Figure 1f); in other cases, sheets of RS cells and variants may occur ('reticular' variant or 'Hodgkin sarcoma'). ${ }^{8}$ Before the availability of immunophenotyping studies, many cases diagnosed as LCHL were LBCL or T-cell lymphomas, often of the anaplastic large cell type. 

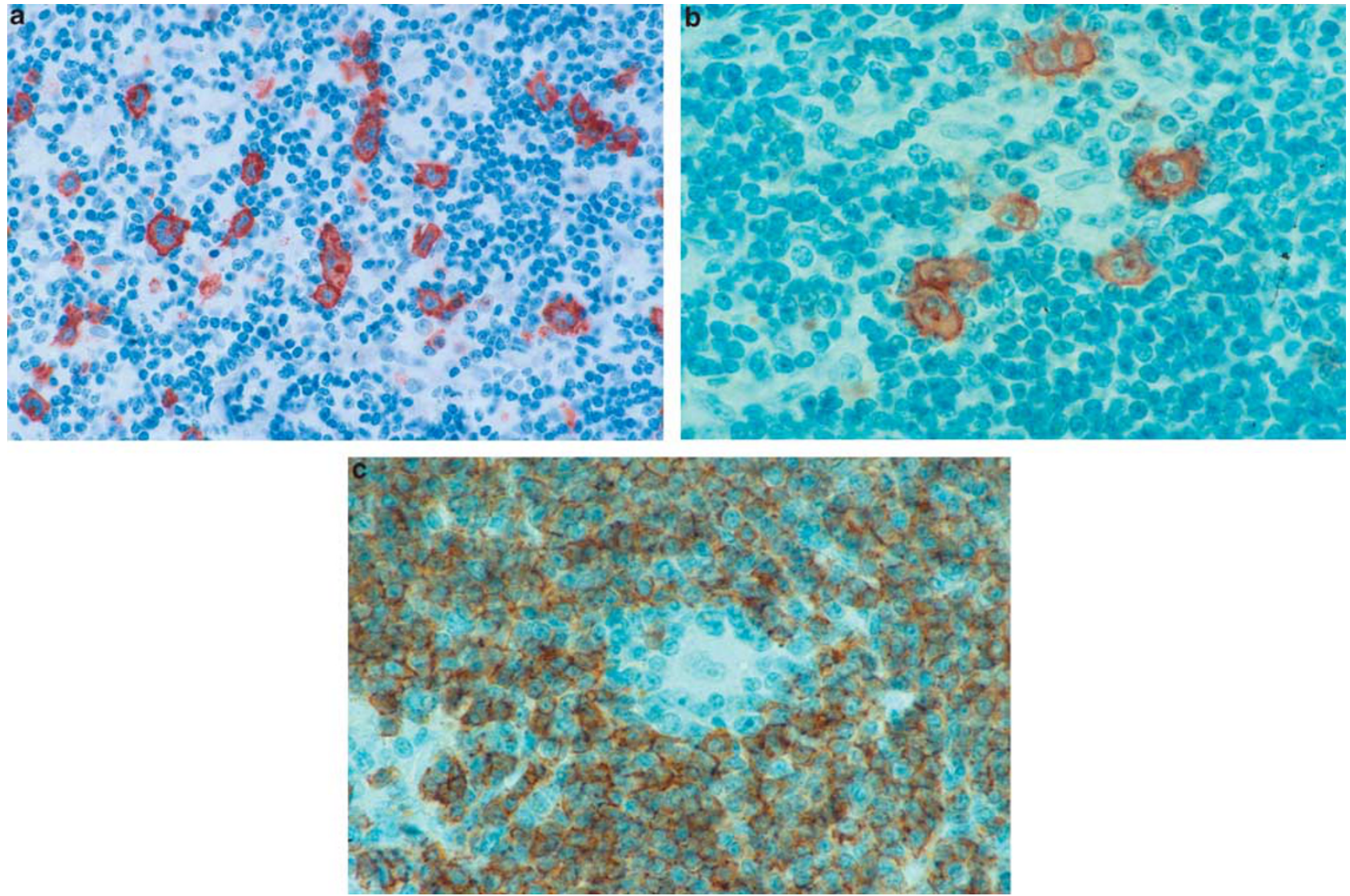

Figure 2 Immunophenotype of classical Hodgkin lymphoma. (a) Lymphocyte-rich classical Hodgkin lymphoma (LRCHL) has ReedSternberg (RS) cells that are CD15 + . (b) The RS cells also express CD30. (c) The RS cells are CD20 - in a background of CD20 + small B lymphocytes.

LDCHL comprises $<1 \%$ of CHL in recent reports. ${ }^{11,12}$ It is seen more commonly in HIV + individuals, and in resource-poor settings than in otherwise healthy, affluent persons. Patients present more commonly with advanced disease at diagnosis and B symptoms than with other subtypes. Response to treatment, progression-free and overall survival are reported to be significantly worse than for other subtypes, but may be improved with very aggressive treatment regimens. ${ }^{11,12}$

\section{Primary mediastinal large B-cell lymphoma}

PMBL is a diffuse LBCL arising in the thymus, from a putative thymic $\mathrm{B}$ cell. ${ }^{13}$ There is a wide morphologic spectrum; a common feature is fine, compartmentalizing sclerosis (Figure 3). The tumor cells are large, typically with pale cytoplasm; the nuclei range from oval to multilobated or pleomorphic, occasionally resembling anaplastic large cells or RS cells. They express most B-cell antigens but lack immunoglobulin, and express CD30 in the majority of the cases, but more weakly than typical CHL; they are usually CD15 - . Bcl6 is expressed in the majority of cases, typically also with IRF4/
Mum1; CD10 is typically absent. Expression of TRAF1 and nuclear REL are characteristic. Most cases express CD23, ${ }^{14,15}$ and this marker has been reported to be useful indistinguishing PMBL from CHL and other diffuse LBCL (DLBCL). Recently, expression of the dendritic cell marker TNFAIP2 by the malignant cells of HL and PMBL has been reported, in contrast to other high-grade lymphomas. ${ }^{16}$ In the absence of a specific marker, the diagnosis typically rests on a combination of clinical features (mediastinal location), morphology and immunophenotype. Rearrangements of BCL2, $B C L 6$ or $M Y C$ genes are uncommon. CGH shows gains at 9p24 and 2p15. Gene expression profiling has shown a unique signature, which shares more features with CHL than with other types of DLBCL. ${ }^{17,18}$ As in HRS cells of CHL, there is constitutive activation of the NFkB and JAK/STAT pathways. Recently, it has been shown that translocations involving the MHC class II transactivator CIITA are found in 38\% of PMBL. ${ }^{4}$

Patients are typically young adults, with a slight female predominance, who present with a rapidly growing, symptomatic mediastinal mass. ${ }^{13}$ Extension to supraclavicular or high subdiaphragmatic lymph nodes may occur, but distant lymph node involvement is rare and should call the diagnosis 

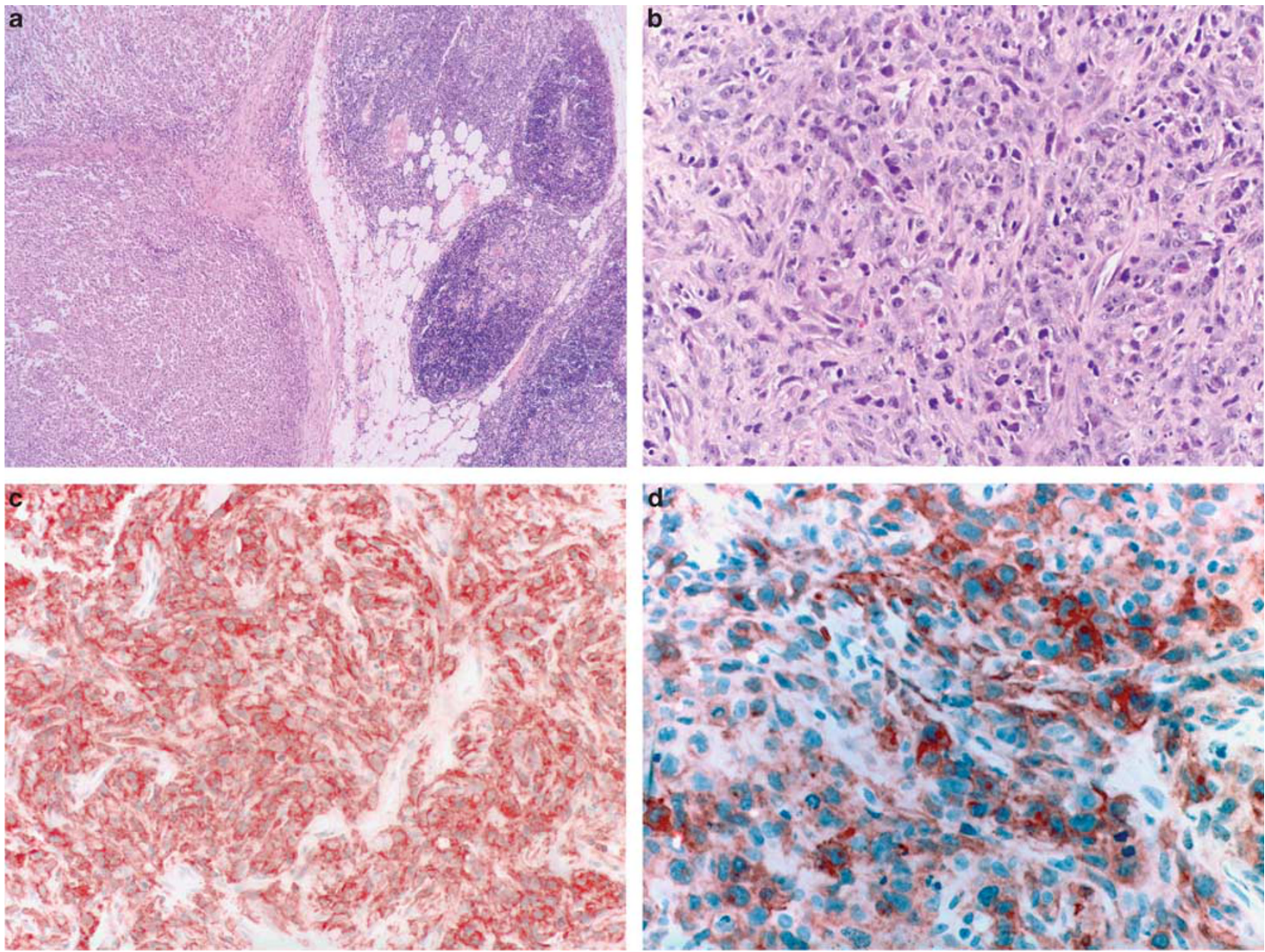

Figure 3 Morphologic and immunophenotypic features of primary mediastinal large B-cell lymphoma (PMBL). (a) At low magnification, the tumor (left) involves the thymus, with a normal thymus remnant seen at right. The tumor has a somewhat nodular architecture, with bands of fibrosis. (b) At high magnification, the tumor cells have oval, vesicular nuclei and one or more basophilic nucleoli. They are embedded in a sclerotic background. (c) The tumor cells express strong CD20 and (d) weak CD30.

into question. Extranodal involvement, including lung, gastrointestinal tract, ovaries, liver, kidneys, CNS and breasts may occur either at the time of diagnosis or relapse.

\section{Gray-zone between HL and PMBL}

Some lymphomas have features of both CHL and DLBCL, particularly NSCHL of the mediastinum and PMBL (Figures 4 and 5). In addition, some patients may have both PMBL and CHL, either synchronously or metachronously (Figure 6). These tumors are most commonly found in the mediastinum, and the term 'mediastinal gray-zone lymphoma' has been used. ${ }^{19}$ Gene expression profiling data suggest that mediastinal LBCL is more closely related to CHL than to most other LBCLs; thus, this phenomenon may reflect true biological overlap between these two diseases. ${ }^{17,18}$ More recently, whole genome sequencing found a gene fusion involving the MHC class II transactivator CIITA in both CHL and PMBL, further suggesting a biological relationship. ${ }^{4}$ Analysis of methylation patterns has shown that mediastinal gray-zone lymphoma has a distinct epigenetic profile intermediate between CHL and PMBL but different from that of diffuse LBCL, suggesting that it is a distinct neoplasm, different from either CHL or PMBL. ${ }^{20}$

The WHO classification in 2008 added a provisional category, B-cell lymphoma, intermediate between DLBCL and CHL. ${ }^{21}$ It is defined as a B-lineage lymphoma that demonstrates overlapping clinical, morphological and/or immunophenotypic features between CHL and DLBCL, especially PMBL.

The tumors are usually composed of confluent sheets of pleomorphic, large tumor cells, often with fibrosis. The cells are often larger and more pleomorphic than typical DLBCL/PMBL, and may resemble lacunar RS cell variants. There may be variation from one area to another, with some areas resembling PMBL and others NSCHL (Figure 4), as well as transitional areas with overlapping features 

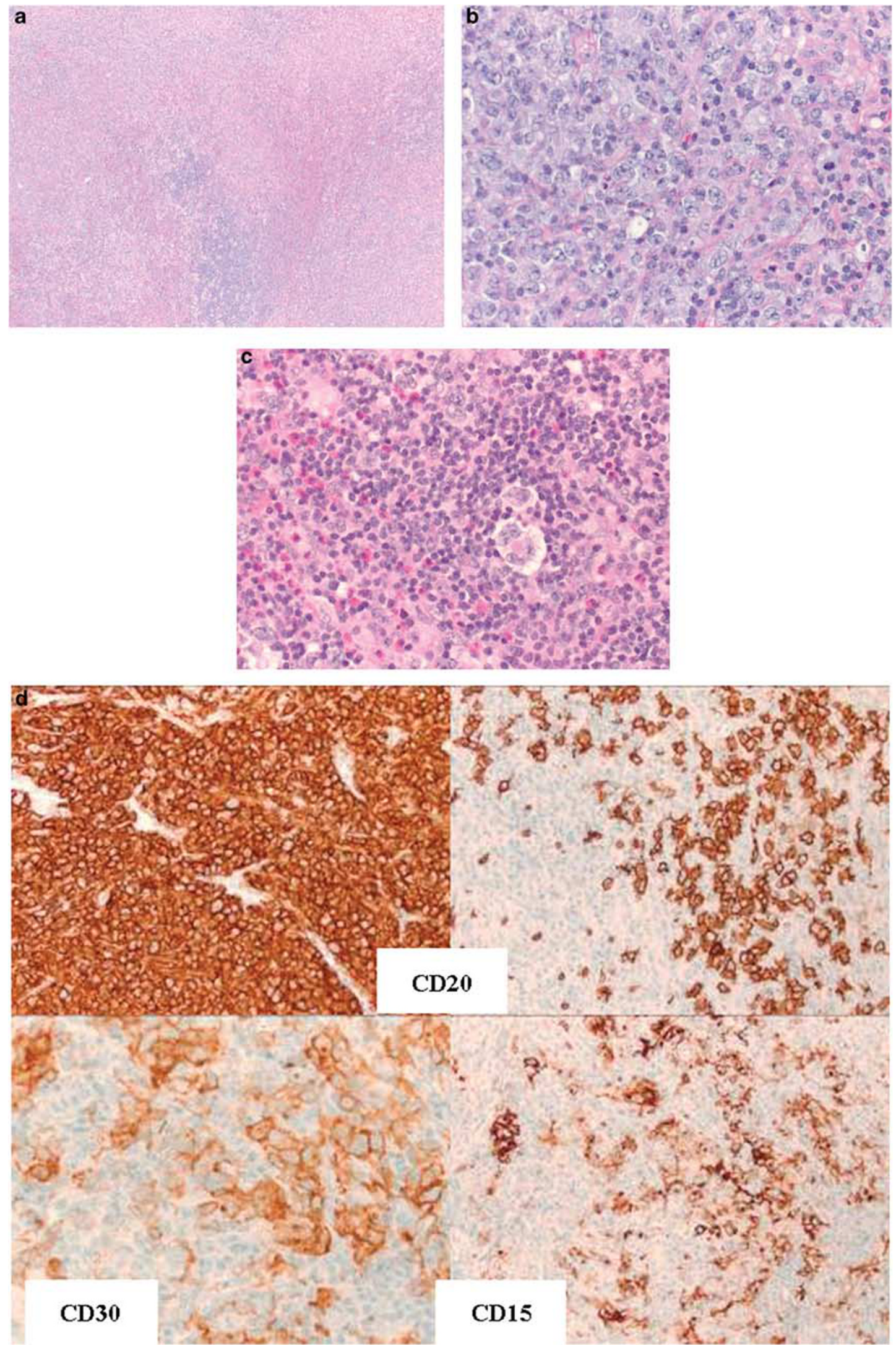
(Figure 5). ${ }^{22}$ The immunophenotype is typically borderline between that of CHL and DLBCL. Tumor cells usually express CD45 and B-cell antigens (CD20, Oct 2, Bob1) as well as CD30 and often CD15 (Figures 4 and 5). They typically do not express immunoglobulin and are EBV $-{ }^{21}$ Suggestions for distinction between CHL and PMBL and gray-zone cases are summarized in Tables 1 and 2 .

These tumors typically present in the mediastinum in young adults, with a male predominance (in contrast to both PMBL and NSCHL); they are clinically more aggressive than either PMBL or NCHL. Optimal treatment is not known and management must be individualized. ${ }^{21,23,24}$

\section{Summary}

The occasional occurrence of both PMBL and CHL in the same patient, together with the existence of the 'gray-zone' cases, suggests the existence of a neoplastic cell with a degree of plasticity, which results not only in variable morphology and immunophenotype of the tumor cells, but in the ability of the cells to influence or be influenced by the microenvironment. In the future, validation of additional markers to distinguish CHL from DLBCL would be useful. An unanswered question is whether 'gray zone' cases are different from composite or sequential cases (Figures 6 and 7). It would also be useful to identify whether there is a way to predict which PMBL cases will relapse as CHL or vice versa. Development of optimal therapy for gray-zone cases is urgently needed. ${ }^{23}$

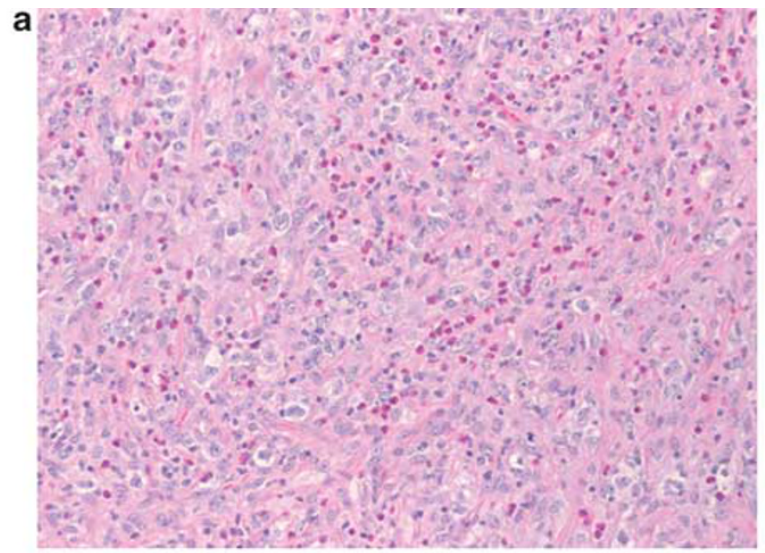

b

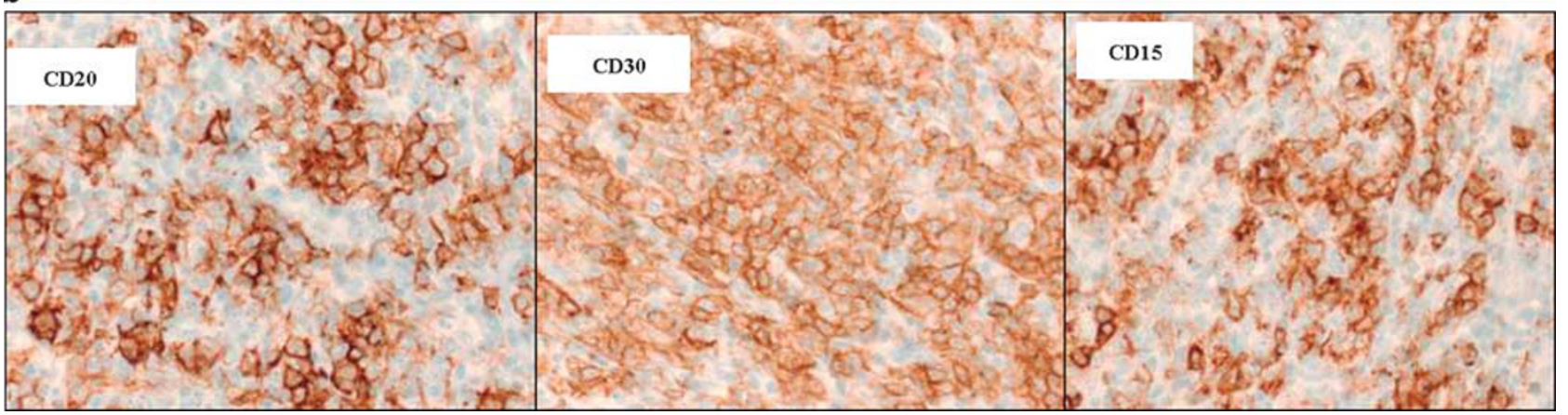

Figure 5 High-grade B-cell lymphoma intermediate between primary mediastinal large B-cell lymphoma (PMBL) and classical Hodgkin lymphoma (CHL). (a) Other sections from the same tumor as in Figure 4 showed features of both CHL and PMBL, with sheets of pleomorphic large cells and many eosinophils. (b) The immunophenotype in these areas is also borderline, with coexpression of CD20, CD30 and CD15.

Figure 4 High-grade B-cell lymphoma intermediate between primary mediastinal large B-cell lymphoma (PMBL) and classical Hodgkin lymphoma (CHL). A 28-year-old woman presented with a mediastinal mass. (a) At low magnification, the predominant pattern resembles PMBL; at the center of the field is an area rich in lymphocytes. (b) At high magnification, the cellular morphology is consistent with PMBL. (c) In the lymphocyte-rich areas, there are numerous eosinophils as well as lacunar variants of Reed-Sternberg (RS) cells. (d) The PMBL-like cells (top left) are strongly CD20 +, whereas the RS-like cells (top right) are predominantly negative. Neoplastic cells in both areas are CD30 + (bottom left); RS-like cells are CD15 +, whereas the PMBL cells are CD15 - . 

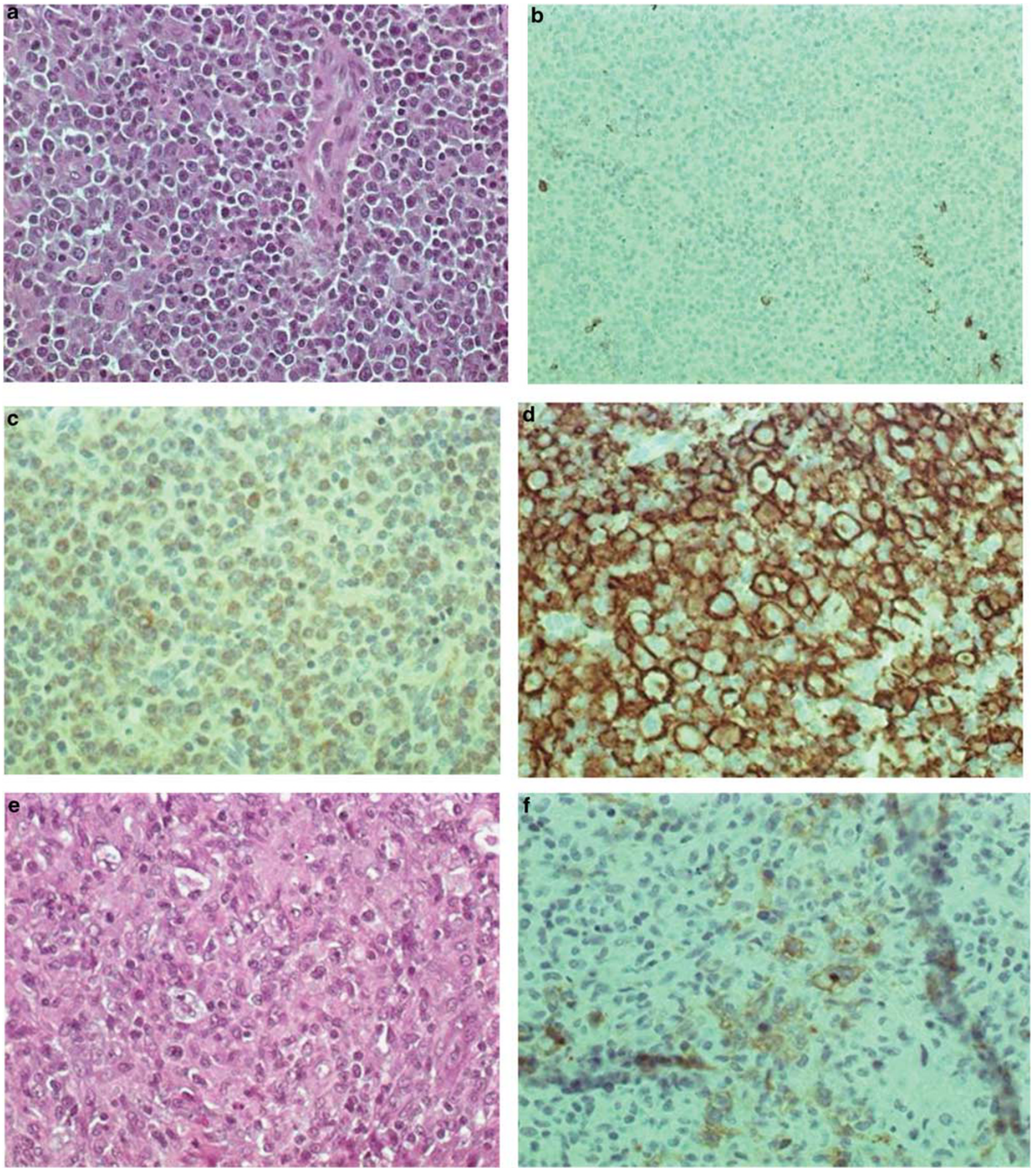

Figure 6 Primary mediastinal large B-cell lymphoma (PMBL) with relapse as classical Hodgkin lymphoma (CHL). A 40-year-old man presented with a mediastinal mass involving the thymus. (a) The tumor is a large-cell lymphoma with no features to suggest CHL (hematoxylin and eosin). (b) The tumor is CD79A + . (c) An unusual feature for PMBL is that it is predominantly CD20 - . (d) There were small collections of CD20 + large cells in the thymic medulla.The tumor cells were strongly Pax5 + and CD15 and CD30 - negative (not shown). The patient achieved complete remission with chemotherapy for PMBL. (e) Four months later, the tumor relapsed in the lung with morphologic features of CHL (hematoxylin and eosin). (f) The tumor cells were CD30 + and CD15 + (not shown) and expressed faint Pax5 (not shown), without other B-cell markers. (b-d, f, immunoperoxidase stain).

lymphoid follicles associated with FDC meshworks (Figure 7). A variable diffuse component may be present and rarely predominate. ${ }^{25}$ Examination of large numbers of sections may be required in some cases to detect foci of nodularity. According to the WHO classification, identification of even one 
Table 1 Diagnostic evaluation of suspected grey-zone lymphoma (borderline between CHL and PMBL)

Morphology

All areas borderline between CHL and PMBL

Gradation or admixture of areas resembling CHL and others resembling PMBL

'Composite lymphoma'-discrete, separate areas of CHL and $\mathrm{PMBL}^{\mathrm{a}}$

'Discordant lymphoma'-sequential appearance of PMBL and $\mathrm{CHL}^{\mathrm{a}}$

Immunophenotyping panel

CD45, CD20, CD15, CD30, Pax5

CD79a, CD19, Oct2, Bob1, Bcl6

${ }^{\mathrm{a}}$ Composite and discordant cases are excluded from WHO definition but are likely related phenomena.

Table 2 Algorithm for differential diagnosis of CHL, PMBL and grey-zone lymphomas

Features that favor PMBL

Absence of nodularity, no eosinophils

CD45 + CD20 + CD79a + Bcl6 + CD19 + CD30 weak CD15 -

Features that favor CHL

Prominent nodularity, sclerotic bands, eosinophils

CD45 - CD20 - / + Pax5 weak Bcl6 - CD30 + + CD15 + Oct2/ Bob1 -

Features that favor 'grey-zone lymphoma' intermediate PMBL/ CHL

Areas with, lacunar cells, eosinophils, sheets of tumor cells resembling PMBL

$\mathrm{CD} 45+\mathrm{CD} 20+\mathrm{CD} 79 \mathrm{a}+\mathrm{CD} 30++\mathrm{CD} 15+$

Not all cases of CD20 + CHL belong in this category

The more B-cell antigens expressed in a tumor resembling CHL, the more likely it is to be grey-zone

nodule of typical NLPHL is sufficient to exclude the diagnosis of THRLBCL.

A variety of immunoarchitectural patterns have been described, including classic B-cell-rich nodular, nodular with prominent extrafollicular LP cells, T-cell-rich nodular, THRLBCL-like and diffuse B-cell rich. ${ }^{26}$ Recently, cases of NLPHL with prominent interfollicular and diffuse areas containing morphologically atypical $\mathrm{T}$ cells have been described, which may mimic peripheral T-cell lymphoma. ${ }^{27}$

The neoplastic cells express B-cell antigens (CD20, CD79a, Pax5, Oct2, Bob1) and lack CD15 and CD30 (weak CD30 may rarely be expressed); immunoglobulin heavy and light chains are typically expressed, and IgD may be seen, particularly in cases affecting young males. The tumor cells are typically Bcl6 + and IRF4/Mum1 is usually but not always negative. The background lymphocytes in nodular areas are predominantly CD20 + B cells, with smaller numbers of CD3 + T cells immediately surrounding the neoplastic cells; the T cells in these areas express markers of follicular helper T cells, including CD4, CD57, PD1/CD279 and Bcl6.9,25 Diffuse areas tend to be more T-cell rich, and in these areas, whereas CD4 + cells still predominate, CD57 + CD279 + are less frequent and do not form rosettes. ${ }^{9}$ By flow cytometry, increased numbers of CD4 + CD8 + double-positive $\mathrm{T}$ cells are often detected. ${ }^{28}$

IGH genes are clonally rearranged, mutated with ongoing mutations, and functional, BCL6/IGH@ rearrangements have been reported in up to $20 \%$ of the cases, and BCL6 rearrangements with other genes in $48 \% .{ }^{25}$ CGH from microdissected CD20 + malignant cells showed recurrent genomic imbalances (average 10.8 per case), involving all chromosomes including 1, 2q, 3, 4q, 5q, 6, 8q, 11q, $12 \mathrm{q}$ and $\mathrm{X}$, and loss of chromosome 17, in 36.8$68.4 \%$ of the cases. ${ }^{29}$ Gains of $2 q, 4 q, 5 q, 6$ and $11 q$ are uncommon in NHL. There were frequent gains of chromosome 6q, a region often deleted in NHL.

NLPHL comprises about $5 \%$ of HL; it may occur at any age, but most often between the ages of 30 and 50 years; it is the most common subtype to occur in pre-adolescent children. There is a strong male predominance. Most cases present as isolated peripheral lymph nodes. The clinical course is indolent, with most patients cured with resection and/or local radiation; late relapses may occur, but are typically localized and responsive to therapy. A recent retrospective study suggested that treatment with ABVD might improve outcomes compared with radiation or observation. ${ }^{30,31}$

\section{T-cell/histiocyte-rich LBCL}

THRLBCL is a LBCL in which only scattered malignant cells are present in a background of lymphocytes, usually with single or small clusters of histiocytes (Figure 8). ${ }^{32}$ The tumor cells may resemble the LP cells of NLPHL, but are more variable in size and shape, or may resemble centroblasts or immunoblasts or even HRS cells. They express B-cell-associated antigens, often EMA and Bcl6, lack CD15 and CD30, and are EBVnegative, but do not otherwise appear to have distinctive immunophenotypic or genetic features. IG genes are rearranged and mutated, with ongoing mutations, consistent with germinal center B cells. ${ }^{32}$ CGH on microdissected cells shows genomic imbalances in all cases (average of 4.7), most often gain of Xq, 4q13q28, Xp21p11 and 18q21, and loss of $17 \mathrm{p}^{33}$ Gene expression profiling studies have identified a subgroup of DLBCL with a high host immune response (HR) signature and frequent spleen and bone marrow involvement, which includes cases of THRLBCL. ${ }^{34}$

Patients are typically middle aged, with a male predominance, and typically have advanced disease with involvement of lymph nodes, liver, spleen and often bone marrow. The response to conventional 

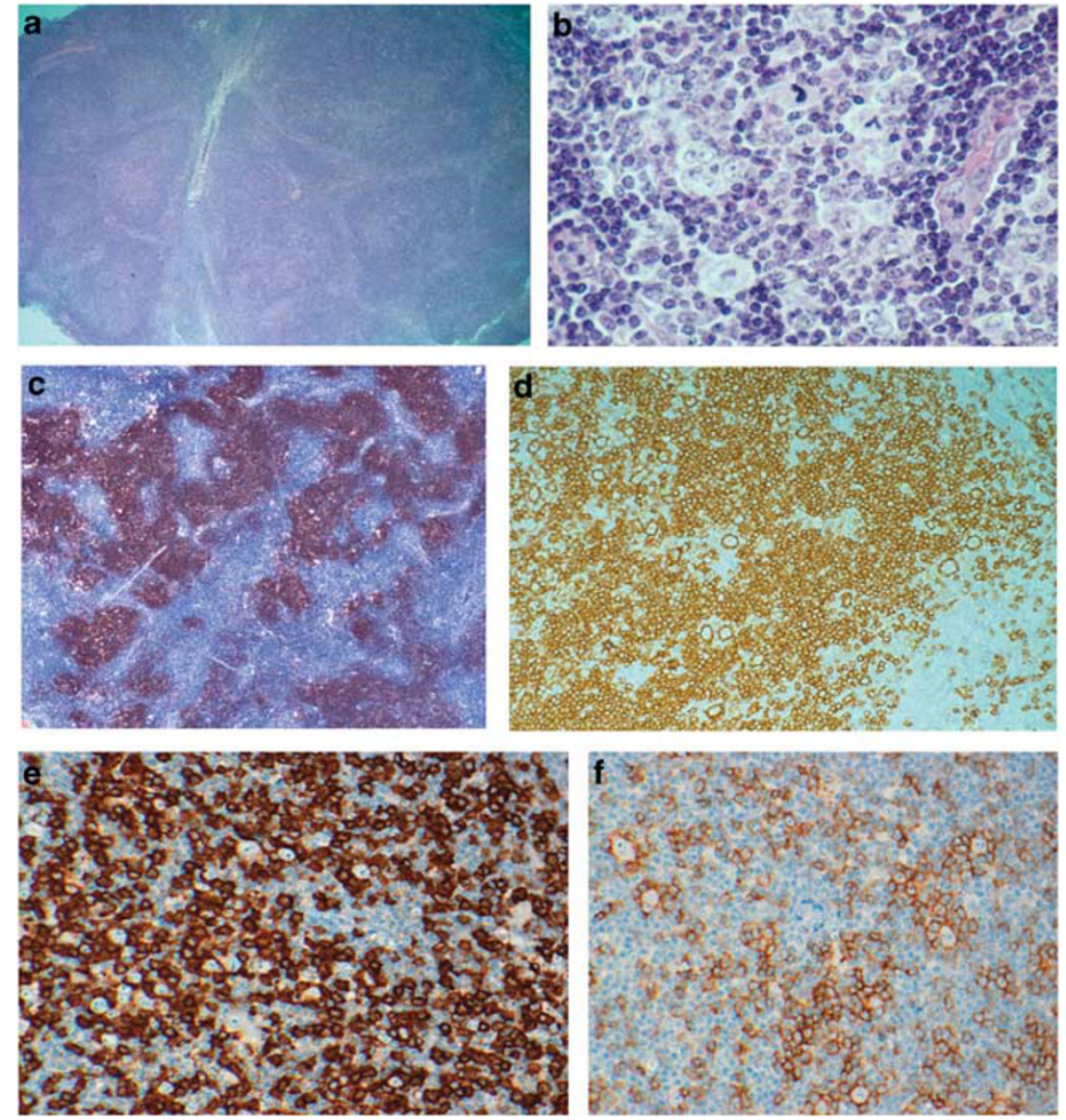

Figure 7 Morphologic and immunophenotypic features of nodular lymphocyte predominant Hodgkin lymphoma (NLPHL). (a) At low magnification NLPHL is characterized by large, poorly circumscribed nodules. (b) The large neoplastic cells have multilobated nuclei, and are known as LP or 'popcorn' cells. (c) The neoplastic cells are found in a background of lymphoid follicles associated with CD21 + follicular dendritic cell (FDC) meshworks. (d) The nodules are B-cell-rich, as shown with a CD20 stain, and the neoplastic cells are also $\mathrm{CD} 20+$. Note the CD20 - T cells surrounding the tumor cells, many of which are CD57 + (e) and CD279/PD1 + (f). (a, b, hematoxylin and eosin; c-f, IHC for CD21, CD20, CD57, CD279, respectively).

chemotherapy is reported to be poor. ${ }^{32}$ However, a recent study found no difference in survival between cases with the HR signature and the other genetically defined subtypes of DLBCL. ${ }^{34}$

\section{Borderline between NLPHL and DLBCL}

Transformation of NLPHL to DLBCL occurs in about $5 \%$ of the cases. The DLBCL may consist of typical LP cells, but usually resembles other DLBCLs. In most cases, a clonal relationship between the LP and the DBCL has been shown by molecular genetic analysis. Particularly in relapsed cases of NLPHL, the pattern may become partly or entirely diffuse, and distinction between diffuse areas of LPHL and THRLBCL may be impossible (Figure 9). Pathologists vary in their interpretation of this phenomenon; some will make a diagnosis of relapse of NLPHL with a diffuse pattern, while others may make a diagnosis of progression to THRLBCL. In the 2008 WHO classification, it was agreed that the diagnosis of THRLBCL should be restricted to primary cases, and that occurrence or relapse of NLPHL with a partially or entirely diffuse pattern should be called either diffuse LPHL or 'NLPHL, THRLBCL-like'. ${ }^{25}$

Distinction of de novo THRLBCL from NLPHL with a predominantly diffuse pattern can be difficult (Table 3). Clinical features, such as young age and isolated peripheral lymphadenopathy (cervical, axillary and inguinal) should give rise to a high index of suspicion for NLPHL over THRLBCL, and prompt careful evaluation of large numbers of sections to look for nodular areas. Neither the morphologic features nor the immunophenotype of the neoplastic cells is definitive in this distinction, although a lack of Bcl6 and expression of IRF4/ MUM1 would favor THRLBCL. It is mainly the background population that is important. Immunophenotyping should include both T-cell and B-cell markers as well as FDC antigens (CD21, CD23 and CD35); staining for CD4, CD8, CD57 and CD279 may also be helpful. The presence of large numbers of $\mathrm{B}$ 

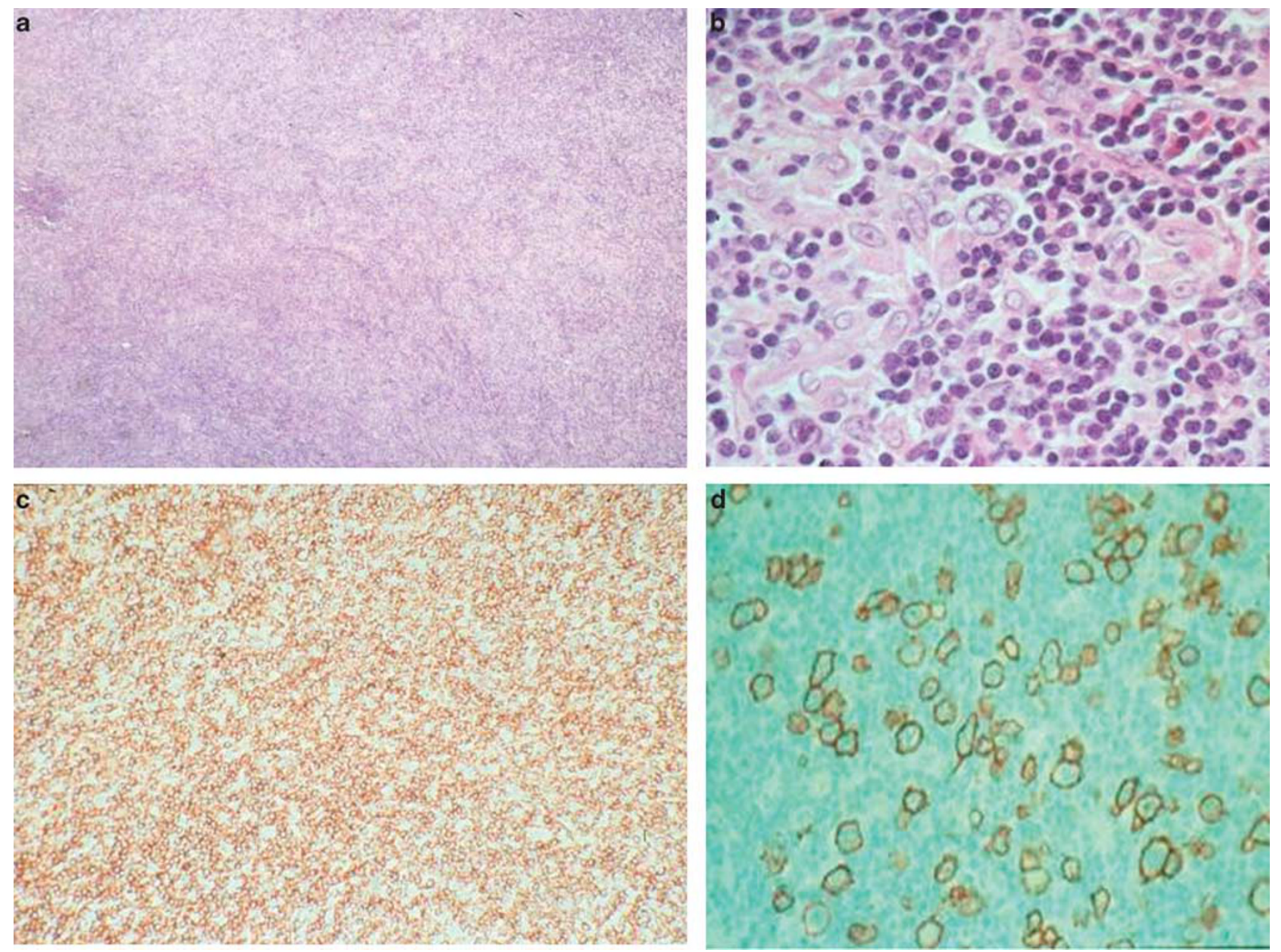

Figure 8 T-cell/histiocyte-rich large B-cell lymphoma (THRLBCL). (a) At low magnification there is a diffuse pattern with a predominance of lymphocytes, with small clusters of histiocytes. (b) There are scattered large neoplastic cells (a, b, hematoxylin and eosin). (c) The background lymphocytes are predominantly CD3 $+\mathrm{T}$ cells. (d) The large cells are CD20 +. (c, d, immunoperoxidase stain).

cells, even in a diffuse distribution, argues strongly against a diagnosis of THRLBCL. Even focal detection of B-cell nodules associated with FDC supports the diagnosis of NLPHL. Bone marrow involvement, while uncommon, may occur in NLPHL and in this location, the background is typically T-cell rich. Thus, distinction between NLPHL and THRLBCL in the bone marrow may not be possible.

Genetic features show some similarities and some differences between NLPHL and THRLBCL. ${ }^{29,33}$ Both have rearranged, mutated IGH genes with ongoing mutations. Partial gain of $4 \mathrm{q}$, a rare change in lymphoma, is frequent in both. However, the number of genomic imbalances (average of 4.7 in T/HRBCL versus 10.8 in LPHL) and their distribution (usually 15 in T/HRBCL versus 6-22 in LPHL) was different. The fact that NLPHL has more complex genetic abnormalities than de novo THRLBCL makes it unlikely that de novo THRLBCL is a form of progression of NLPHL. Finally, BCL6 translocations, which are relatively common in NLPHL, have not been reported as a recurrent abnormality in THRLBCL. ${ }^{25,32}$

\section{Summary}

The tendency of NLPHL to lose its B-cell-rich follicular background, either focally at the time of the diagnosis, or with disease progression, becoming diffuse and T-cell rich, suggests a degree of plasticity of this tumor cell as well-in some circumstances requiring or acquiring a background of an altered B-cell follicle for survival, and in others able to grow and survive in a diffuse T-cell-rich background. Although definitive evidence that the T-cell-rich cases have a worse prognosis is lacking, it is possible that the ability of the cell to survive in the absence of the follicular environment is due to secondary genetic alterations that result in a more aggressive phenotype. The available evidence suggests that de novo THRLBCL is distinct from progressed NLPHL resembling THRLBCL.

In the future, the clinical significance of diffuse areas and/or progression to a diffuse pattern in NLPHL needs to be better defined. This is a rare complication of a rare disease, so that a multi- 

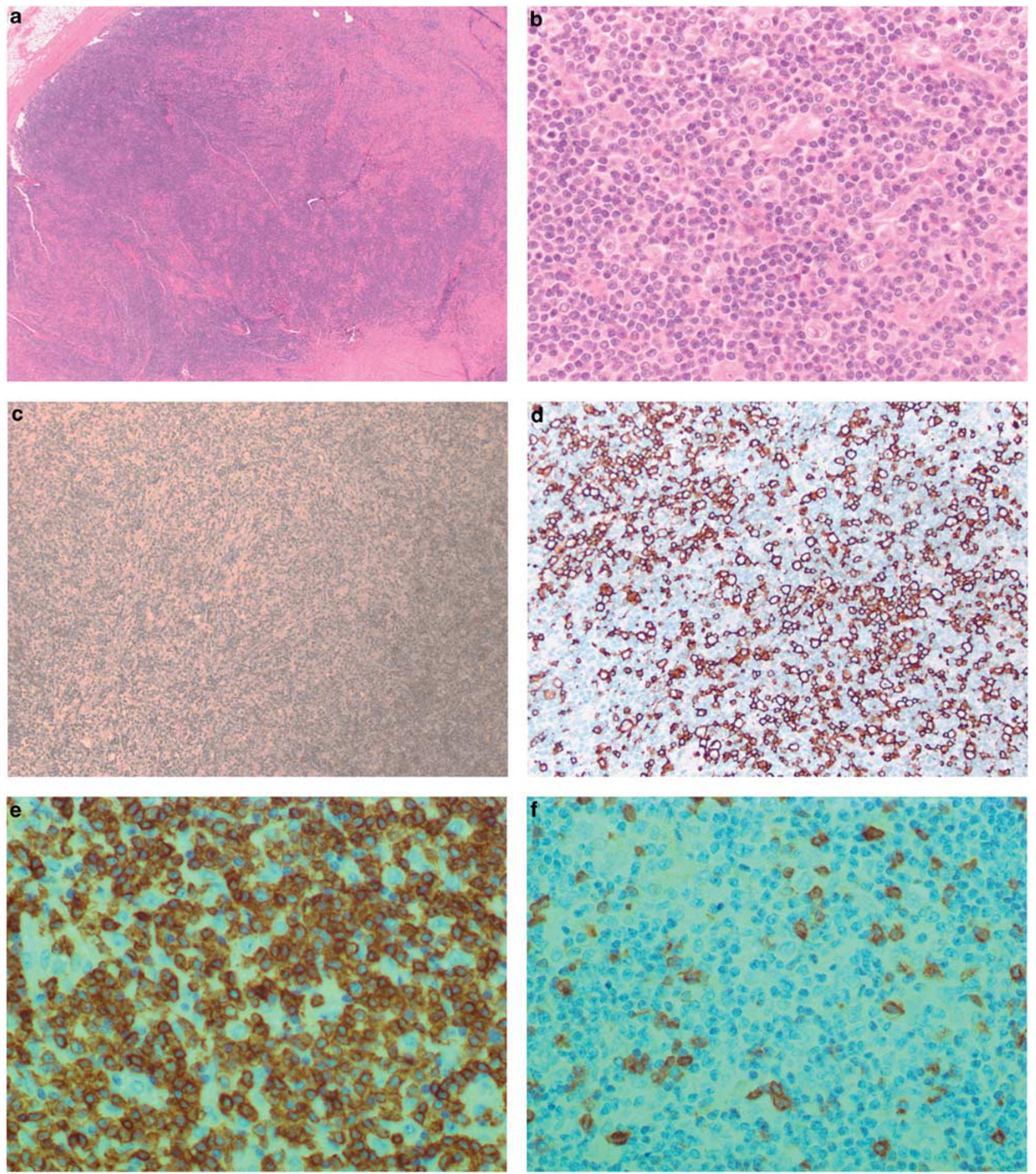

Figure 9 Nodular lymphocyte predominant Hodgkin lymphoma (NLPHL) with a predominantly diffuse pattern. (a) The pattern is diffuse, and there are clusters of epithelioid histiocytes evident at low magnification (hematoxylin and eosin (H\&E)). (b) At high magnification, large neoplastic cells are present in a background of lymphocytes (H\&E). (c) With immunohistochemistry to CD21, no follicular dendritic cell (FDC) meshworks are seen. (d) The large cells are CD20 + but so are many background small lymphocytes. (e) The majority of small lymphocytes are CD3 + T cells. (f) Many T cells are CD57 + . In other areas, a typical nodular B-cell-rich pattern was seen (not shown).

institutional study with review of slides and long clinical follow-up will likely be required. Finally, better immunophenotypic and/or genetic markers would be helpful, both to allow better differentiation between NLPHL and TCRBCL, and to show whether or not they are related diseases. 
Table 3 Algorithm for differential diagnosis of NLPHL diffuse and THRLBCL

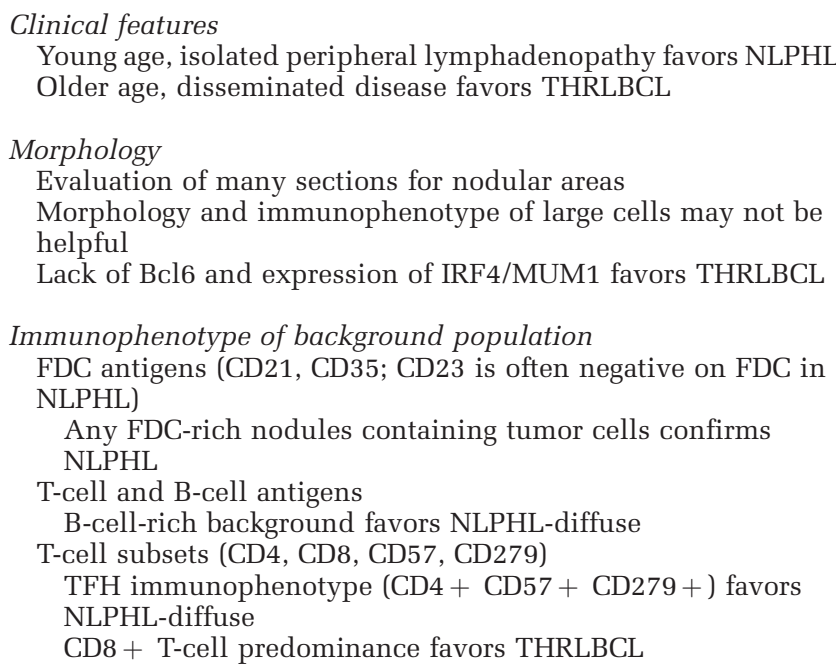

\section{Disclosure/conflict of interest}

The author declares no conflict of interest.

\section{References}

1 Harris NL, Jaffe ES, Stein H, et al. A revised EuropeanAmerican classification of lymphoid neoplasms: a proposal from the International Lymphoma Study Group. Blood 1994;84:1361-1392.

2 Stein H. Hodgkin lymphoma-introduction, In: Swerdlow SH, Campo E, Harris NL, Jaffe ES, Pileri S, Stein H, Thiele J, Vardiman J (eds). WHO Classification of Tumours of Haematopoietic and Lymphoid Tissues, 4th edn. IARC: Lyon; 2008, pp 322.

3 Stein H, Delsol G, Pileri SA, et al. Classical Hodgkin lymphoma, introduction, In: Swerdlow SH, Campo E, Harris NL, Jaffe ES, Pileri S, Stein H, Thiele J, Vardiman J (eds). WHO Classification of Tumours of Haematopoietic and Lymphoid Tissues, 4th edn. IARC: Lyon; 2008, pp 326-330.

4 Steidl C, Shah SP, Woolcock BW, et al. MHC class II transactivator CIITA is a recurrent gene fusion partner in lymphoid cancers. Nature 2011;471:377-381.

5 Stein H, von Wasielweski R, Poppema S, et al. Nodular sclerosis classical Hodgkin lymphoma, In: Swerdlow SH, Campo E, Harris NL, Jaffe ES, Pileri S, Stein H, Thiele J, Vardiman J (eds). WHO Classification of Tumours of Haematopoietic and Lymphoid Tissues, 4th edn. IARC: Lyon; 2008, pp 330.

6 Weiss LM, von Wasielweski R, Delsol G, et al. Mixed cellularity classical Hodgkin lymphoma, In: Swerdlow SH, Campo E, Harris NL, Jaffe ES, Pileri S, Stein H, Thiele J, Vardiman J (eds). WHO Classification of Tumours of Haematopoietic and Lymphoid Tissues, 4th edn. IARC: Lyon; 2008, pp 331.

7 Anagnostopoulos I, Isaacson P, Stein H. Lymphocyterich classical Hodgkin lymphoma, In: Swerdlow SH, Campo E, Harris NL, Jaffe ES, Pileri S, Stein H, Thiele J,
Vardiman J (eds). WHO Classification of Tumours of Haematopoietic and Lymphoid Tissues, 4th edn. IARC: Lyon; 2008, pp 332-333.

8 Benharroch D, Stein H, Peh S-C. Lymphocyte-depleted classical Hodgkin lymphoma, In: Swerdlow SH, Campo E, Harris NL, Jaffe ES, Pileri S, Stein H, Thiele J, Vardiman J (eds). WHO Classification of Tumours of Haematopoietic and Lymphoid Tissues, 4th edn. IARC: Lyon; 2008, pp 334.

9 Nam-Cha SH, Roncador G, Sanchez-Verde L, et al. PD1, a follicular T-cell marker useful for recognizing nodular lymphocyte-predominant Hodgkin lymphoma. Am J Surg Pathol 2008;32:1252-1257.

10 Nam-Cha SH, Montes-Moreno S, Salcedo MT, et al. Lymphocyte-rich classical Hodgkin's lymphoma: distinctive tumor and microenvironment markers. Mod Pathol 2009;22:1006-1015.

11 Shimabukuro-Vornhagen A, Haverkamp H, Engert A, et al. Lymphocyte-rich classical Hodgkin's lymphoma: clinical presentation and treatment outcome in 100 patients treated within German Hodgkin's Study Group trials. J Clin Oncol 2005;23:5739-5745.

12 Klimm B, Franklin J, Stein H, et al. Lymphocytedepleted classical Hodgkin's lymphoma: a comprehensive analysis from the German Hodgkin study group. J Clin Oncol 2011;29:3914-3920.

13 Gaulard P, Harris NL, Pileri S, et al. Primary mediastinal (thymic) large B-cell lymphoma, In: Swerdlow SH, Campo E, Harris NL, Jaffe ES, Pileri S, Stein H, Thiele J, Vardiman J (eds). WHO Classification of Tumours of Haematopoietic and Lymphoid Tissues, 4th edn. IARC: Lyon; 2008, pp 250-251.

14 Calaminici M, Piper K, Lee AM, et al. CD23 expression in mediastinal large B-cell lymphomas. Histopathology 2004;45:619-624.

15 Salama ME, Rajan Mariappan M, Inamdar K, et al. The value of CD23 expression as an additional marker in distinguishing mediastinal (thymic) large B-cell lymphoma from Hodgkin lymphoma. Int J Surg Pathol 2010;18:121-128.

16 Kondratiev S, Duraisamy S, Unitt CL, et al. Aberrant expression of the dendritic cell marker TNFAIP2 by the malignant cells of Hodgkin lymphoma and primary mediastinal large B-cell lymphoma distinguishes these tumor types from morphologically and phenotypically similar lymphomas. Am J Surg Pathol 2011;35: 1531-1539.

17 Rosenwald A, Wright G, Leroy K, et al. Molecular diagnosis of primary mediastinal B cell lymphoma identifies a clinically favorable subgroup of diffuse large B cell lymphoma related to Hodgkin lymphoma. J Exp Med 2003;198:851-862.

18 Savage KJ, Monti S, Kutok JL, et al. The molecular signature of mediastinal large B-cell lymphoma differs from that of other diffuse large B-cell lymphomas and shares features with classical Hodgkin lymphoma. Blood 2003;102:3871-3879.

19 Traverse-Glehen A, Pittaluga S, Gaulard P, et al. Mediastinal gray zone lymphoma: the missing link between classic Hodgkin's lymphoma and mediastinal large B-cell lymphoma. Am J Surg Pathol 2005;29:1411-1421.

20 Eberle FC, Rodriguez-Canales J, Wei L, et al. Methylation profiling of mediastinal gray zone lymphoma reveals a distinctive signature with elements shared by classical Hodgkin's lymphoma and primary mediastinal large B-cell lymphoma. Haematologica 2011; 96:558-566. 
21 Jaffe E, Stein H, Swerdlow S, et al. B-cell lymphoma, unclassifiable, with features intermediate between diffuse large B-cell lymphoma and classical Hodgkin lymphoma, In: Swerdlow S, Campo E, Harris NL, et al. (eds). WHO Classification of Tumours of Haematopoietic and Lymphoid Systems, 4th edn. IARC: Lyon; 2008, pp 267-268.

22 Jaffe E, Stein H, Swerdlow S, et al. B-cell lymphoma, unclassificable, with features intermediate between diffuse large B-cell lymphoma and classical Hodgkin lymphoma, In: Swerdlow SH, Campo E, Harris NL, Jaffe ES, Pileri S, Stein H, Thiele J, Vardiman J (eds). WHO Classification of Tumours of Haematopoietic and Lymphoid Systems, 4th edn. IARC: Lyon; 2008, pp 267-268.

23 Grant C, Dunleavy K, Eberle FC, et al. Primary mediastinal large B-cell lymphoma, classic Hodgkin lymphoma presenting in the mediastinum, and mediastinal gray zone lymphoma: what is the oncologist to do? Curr Hematol Malig Rep 2011;6:157-163.

24 Oschlies I, Burkhardt B, Salaverria I, et al. Clinical, pathological and genetic features of primary mediastinal large B-cell lymphomas and mediastinal gray zone lymphomas in children. Haematologica 2011;96: 262-268.

25 Poppema S, Delsol G, Pileri SA, et al. Nodular lymphocyte predominant Hodgkin lymphoma, In: Swerdlow SH, Campo E, Harris NL, Jaffe ES, Pileri S, Stein H, Thiele J, Vardiman J (eds). WHO Classification of Tumours of Haematopoietic and Lymphoid Tissues, 4th edn. IARC: Lyon, 2008.

26 Fan Z, Natkunam Y, Bair E, et al. Characterization of variant patterns of nodular lymphocyte predominant hodgkin lymphoma with immunohistologic and clinical correlation. Am J Surg Pathol 2003;27:1346-1356.

27 Sohani AR, Jaffe ES, Harris NL, et al. Nodular lymphocyte-predominant Hodgkin lymphoma with atypical T cells: a morphologic variant mimicking peripheral T-cell lymphoma. Am J Surg Pathol 2011;35:1666-1678.

28 Rahemtullah A, Reichard KK, Preffer FI, et al. A double-positive CD4 + CD8 + T-cell population is commonly found in nodular lymphocyte predominant Hodgkin lymphoma. Am J Clin Pathol 2006;126: 805-814.

29 Franke S, Wlodarska I, Maes B, et al. Lymphocyte predominance Hodgkin disease is characterized by recurrent genomic imbalances. Blood 2001;97: 1845-1853.

30 Savage KJ, Skinnider B, Al-Mansour M, et al. Treating limited-stage nodular lymphocyte predominant Hodgkin lymphoma similarly to classical Hodgkin lymphoma with ABVD may improve outcome. Blood 2011;118:4585-4590.

31 Mauz-Korholz C, Gorde-Grosjean S, Hasenclever D, et al. Resection alone in 58 children with limited stage, lymphocyte-predominant Hodgkin lymphoma-experience from the European network group on pediatric Hodgkin lymphoma. Cancer 2007;110:179-185.

32 De Wolf-Peeters C, Delabie J, Campo E, et al. T-cell/ histiocyte-rich large B-cell lymphoma, In: Swerdlow SH, Campo E, Harris NL, Jaffe ES, Pileri S, Stein H, Thiele J, Vardiman J (eds). WHO Classification of Tumours of Haematopoietic and Lymphoid Tissues, 4th edn. IARC: Lyon; 2008, pp 238-239.

33 Franke S, Wlodarska I, Maes B, et al. Comparative genomic hybridization pattern distinguishes T-cell/ histiocyte-rich B-cell lymphoma from nodular lymphocyte predominance Hodgkin's lymphoma. Am J Pathol 2002;161:1861-1867.

34 Monti S, Savage KJ, Kutok JL, et al. Molecular profiling of diffuse large B-cell lymphoma identifies robust subtypes including one characterized by host inflammatory response. Blood 2005;105:1851-1861. 